\title{
Early evolution of Mars with mantle compositional stratification or hydrothermal crustal cooling
}

\author{
E. M. Parmentier ${ }^{1}$ and M. T. Zuber ${ }^{2}$ \\ Received 28 October 2005; revised 11 September 2006; accepted 9 October 2006; published 16 February 2007.
}

[1] Analysis of Martian gravity and topography implies that crustal thickness variations created in the earliest evolution of planet have persisted to the present day. Relaxation of crustal thickness variations due to lower crustal flow by thermally activated creep is strongly temperature-dependent and so, for particular crustal rheology and thickness, provides a constraint on thermal evolution. Previous models have assumed that the heat flux from the mantle, which controls lower crustal temperatures, simply reflects radiogenic heat production. However, global thermal evolution models in which the mantle cools by solid-state thermal convection indicate that the additional contribution to near-surface heat flux due to secular cooling significant increases lower crustal temperatures. With, a 50-km-thick crust, and a wet diabase rheology, these higher temperatures allow crustal rock to flow fast enough to relax crustal thickness variations during the first $\sim 750$ Myr of evolution. Previous studies have not considered the presence of an impact-brecciated crustal layer that would almost certainly be present on early Mars and could have a significant influence on crustal temperatures. The presence of a dry brecciated crustal layer several kilometers thick would significantly increase the temperature of the lower crust resulting in more rapid relaxation of crustal thickness variations. If the porosity of a brecciated crust is water saturated, then the effect of such a layer is greatly reduced. However, if water is present, cooling of the upper crust by groundwater convection in a deeper impact-fractured crustal layer with modest permeabilities could reduce lower crustal temperatures enough to explain the preservation of ancient crustal thickness variations. A cooler lower crust is also possible if solid-state thermal convection in the mantle is inhibited by a stable compositional stratification resulting from the fractional solidification of an early magma ocean.

Citation: Parmentier, E. M., and M. T. Zuber (2007), Early evolution of Mars with mantle compositional stratification or hydrothermal crustal cooling, J. Geophys. Res., 112, E02007, doi:10.1029/2005JE002626.

\section{Introduction}

[2] Analysis of gravity and topography data for Mars show the presence of lateral crustal thickness variations, even beneath features of great age [Zuber et al., 2000; McKenzie et al., 2002; McGovern et al., 2002, 2004]. Variations in the thickness of a uniform density crust cause lateral pressure gradients that generate flow in a ductile lower crust. Flow from areas of larger to smaller crustal thickness evens out the thickness of the crust over time. The largest scale, and perhaps the longest lived, variation present is the north-south crustal thickness variation [Zuber et al., 2000]. At an only slightly smaller scale, the Tharsis rise appears to be a large center of basaltic volcanism, that began forming early in the evolution of Mars [Phillips et al.,

\footnotetext{
${ }^{1}$ Department of Geological Sciences, Brown University, Providence, Rhode Island, USA.

${ }^{2}$ Department of Earth, Atmospheric, and Planetary Sciences, Massachusetts Institute of Technology, Cambridge, Massachusetts, USA.

Copyright 2007 by the American Geophysical Union. 0148-0227/07/2005JE002626\$09.00
}

2001], with topography supported largely by thickened crust. Other major topographic features such as large basins, like Hellas, while apparently younger than the earliest Tharsis volcanism, also preserve crustal thickness variations. In this study, we explore the implications that the survival of such crustal thickness variations would have for the thermal evolution of Mars.

[3] Previous studies [Zuber et al., 2000] have argued that the preservation of crustal thickness variations places constraints on the thickness of the crust through the temperature dependence of crustal viscosity. Nimmo and Stevenson [2001] investigated constraints on crustal thickness using steady state thermal evolution models which assumed a heat flux at the base of the crust equal to the radiogenic heat production in the mantle. With a dry diabase flow law, they found that crustal thicknesses as large as $100 \mathrm{~km}$ would allow the preservation of crustal thickness variations. Since the rheology of the lower crust is likely to be very strongly temperature-dependent, calculated crustal relaxation rates depend sensitively on both mantle thermal evolution and crustal rates of heat transfer. 
(a)

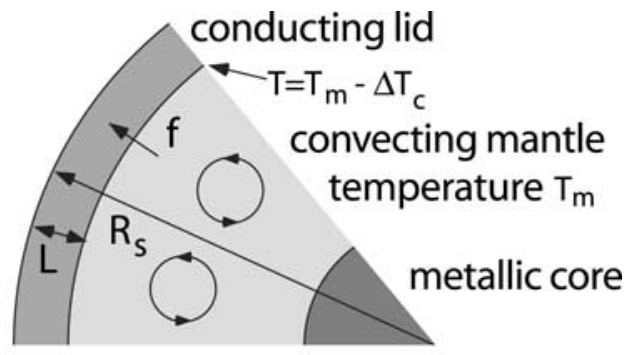

(b)

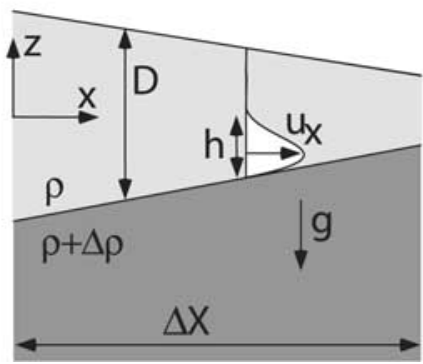

Figure 1. (a) Schematic showing solid state convective mantle cooling beneath a conducting lid that develops in response to high creep viscosity at low temperatures. Other quantities shown are discussed in the text. (b) Schematic illustration of horizontal flow in the lower crust in response to pressure gradients resulting from variations in crustal thickness. Increasing temperature with depth and a strongly temperature-dependent rheology result in flow restricted to a layer of thickness $h$ at the bottom of the crust.

[4] We have formulated thermal evolution models that include secular cooling as well as radiogenic heating. These models treat the strong temperature dependence of mantle flow by thermally activated creep and the presence of a conductive lid above a convecting interior using the scaling laws of Grasset and Parmentier [1998]. Our results [Parmentier and Zuber, 2001, 2002] indicate that the added heat flux from secular cooling of the core and mantle results in significantly higher temperatures at the base of crust. Higher temperatures near the base of the crust allow more rapid flow in response to crustal thickness variations and more rapid relaxation of these variations. For initial mantle potential temperatures for subsolidus cooling in the range of $1500^{\circ}-1800^{\circ} \mathrm{C}$ and a 50 -km-thick crust, a dry diabase rheology based on laboratory flow laws allows the preservation of global-scale crustal thickness variations, except for cases with large amounts of radioactive heating in the crust. For a wet crustal rheology significant relaxation of crustal thickness variations can occur over the first $\sim 750 \mathrm{Myr}$ of evolution (the Noachian epoch).

[5] Lower crustal temperatures may be significantly affected by the presence of a porous, brecciated, lowconductivity layer (regolith) and a deeper layer of fractured crust beneath, as has been identified within the Lunar crust. While the details can be debated, such a layer is almost certainly present, and its effect has not been included in earlier studies. If this brecciated layer is dry, thermal conductivity can be significantly reduced, and layer thick- nesses exceeding only a kilometer significantly enhances the relaxation of crustal thickness variations.

[6] With a wet crustal rheology, and especially so with an impact-brecciated surface layer present, some mechanism is needed to reduce lower crustal temperatures and so preserve crustal thickness variations. Two mechanisms are suggested that can reduce the temperatures of the lower crust, thus preserving crustal thickness variations without requiring a crust thinner than that indicated by recent gravity studies [Neumann et al., 2004]. First, hydrothermal circulation of groundwater within a fractured upper crust may enhance the rate of heat transfer, thus reducing the temperature of the lower crust and enhancing the preservation of ancient crustal thickness variations. Alternatively, an initial stable compositional stratification of the mantle [Zaranek and Parmentier, 2002; Elkins-Tanton et al., 2005a], following fractional solidification of a magma ocean and subsequent mantle overturn, may suppress mantle solid-state thermal convection and fractionate heat sources into the deep mantle, thus reducing the heat flux to base of the crust.

\section{Formulation of Thermal Evolution Models}

[7] As in many previous studies, our mantle thermal evolution models are formulated on the basis of energy conservation in which the rate of change of thermal energy in the planetary interior is equated to the difference between the outward heat flux and the rate of radiogenic heat production

$$
\left[\rho c_{p}\right] \frac{d T_{m}}{d t}=-\left(\frac{3}{R_{s}-L}\right) f+H,
$$

where $T_{m}$ is the mantle potential temperature, $H$ is the volumetric heat production, $R_{S}$ is the radius of the planet, and $f$ is the solid-state convective heat flux at the base of a conductive lid, or lithosphere, of thickness $L$, as illustrated in Figure 1. Here $\rho$ and $c_{p}$ are density and heat capacity, respectively, with [] indicating the average value for the planetary interior (see discussion below). Thermally activated creep can be described by a viscosity $\mu$ with an Arrhenius temperature dependence

$$
\mu=\mu\left(T_{m}\right) \exp \left[\frac{Q}{R T_{m}}\left(\frac{T_{m}}{T}-1\right)\right],
$$

where $Q$ is the activation energy and $R$ is the gas constant. The base of the conductive lid is defined by the isotherm $T_{m}-\Delta T_{c}$ at which the viscosity increases by about 1 order of magnitude relative to that in the adiabatic, convecting interior. Then

$$
\Delta T_{c}=2.2 \frac{R T_{m}^{2}}{Q}
$$

Thus the temperature at the base of the lithosphere does not have a fixed value but varies with the mantle potential temperature. The relationship in (3) is strictly valid only for a viscosity that varies exponentially with temperature, and an additional factor that is nearly unity appears for an Arrhenius temperature dependence [Zaranek and Parmentier, 2004]. 
The heat flux provided to the base of the conductive lid by solid state convection is

$$
f=C k\left(\frac{\rho \alpha g}{\mu\left(T_{m}\right) \kappa}\right)^{1 / 3} \Delta T_{c}^{4 / 3}
$$

where $\kappa$ is the thermal diffusivity, $k$ is the thermal conductivity, $g$ is the acceleration of gravity, $\alpha$ is the coefficient of thermal expansion, and $C=0.185$ [Grasset and Parmentier, 1998].

[8] The thermal structure consists of a convecting, adiabatically stratified mantle with a thermal boundary layer just beneath the conductive lid across which heat in the convecting mantle is transferred to the lid. A measure of the thickness of this boundary layer is defined as the depth at the temperature extrapolated to depth along the conductive temperature gradient transporting the heat flux $f$ reaches the potential temperature $T_{m}$

$$
\delta_{c}=\frac{k \Delta T_{c}}{f} .
$$

This idealization for convection in a fluid with strongly temperature-dependent viscosity cooled from above is consistent with both laboratory [Davaille and Jaupart, 1993] and numerical experiments [e.g., Grasset and Parmentier, 1998].

[9] The heat flux out of a cooling and perhaps solidifying core heats the convecting mantle from below. Earlier studies also treat thermal convection in a fluid with a strongly temperature-dependent viscosity heated from below [Solomatov and Moresi, 2000; Reese et al., 1999]. Heating from below results in a thermal boundary layer at the base of the mantle, generating plumes which transfer heat upward into the overlying mantle. As in previous studies, [e.g., Nimmo and Stevenson, 2000], we assume that the heating of the mantle by these plumes can be represented simply by an additional source of volumetric heating. Recent and ongoing studies [Choblet et al., 1999] of thermal convection driven by both volumetric heating and heating from below suggest that this is an excellent approximation. Assuming that the core and mantle cool together during secular cooling, maintaining a nearly constant temperature difference, the heat flux out of a cooling, solidifying core can be represented by a higher effective volumetric heat capacity $\rho c_{p}$ in (1). The bulk heat capacity of the planet, $\left[\rho c_{p}\right]$ in $(1)$, is taken to be the volume weighed average of $\rho c_{p}$ in the mantle and core, using parameter values listed in Table 1. A mantle and core that cool together should be a good approximation if the core is a turbulently convecting liquid. If the core solidifies, then this approximation is valid only if the secular cooling rate is slow compared to the conduction time for heat out of the core. As the core solidifies, the release of latent heat will result in a higher effective heat capacity. We have not included the effect of latent heat since this added complexity would only result in a higher effective heat capacity that would further increase the importance of secular cooling relative to radioactive heating.

[10] Convective heat transfer given by (4) is applied only after a suitably defined Rayleigh number attains a critical
Table 1. Parameter Values Used in Thermal Evolution and Crustal Relaxation Models

\begin{tabular}{llll}
\hline \multicolumn{1}{c}{ Physical Property } & Symbol & \multicolumn{1}{c}{ Units } & \multicolumn{1}{c}{ Value } \\
\hline $\mathrm{U}$ abundance & - & $\mathrm{ppm}$ & 0.026 \\
$\mathrm{Th} / \mathrm{U}$ & - & - & 4 \\
$\mathrm{~K} / \mathrm{U}$ & - & - & $10^{4}$ \\
Mantle density & $\rho$ & $\mathrm{kg} / \mathrm{m}^{3}$ & 3500 \\
Core density & $\rho$ & $\mathrm{kg} / \mathrm{m}^{3}$ & 9000 \\
Mantle specific heat & $c_{p}$ & $\mathrm{~kJ} / \mathrm{kg}^{\circ} \mathrm{C}$ & 1 \\
Core specific heat & $c_{p}$ & $\mathrm{~kJ} / \mathrm{kg}^{\circ}{ }^{\circ} \mathrm{C}$ & 0.8 \\
Thermal conductivity & $k$ & $\mathrm{~W} / \mathrm{m}^{2}-{ }^{\circ} \mathrm{C}$ & 3 \\
Core radius & $R_{c}$ & $\mathrm{~km}$ & 1400 \\
Planet radius & $R_{s}$ & $\mathrm{~km}$ & 3400 \\
Surface temperature & $T_{s}$ & ${ }^{\circ} \mathrm{K}$ & $220-273$ \\
Mantle reference viscosity at & $\mu\left(T_{m}\right)$ & $\mathrm{Pa}-\mathrm{s}$ & 10 \\
$\quad T_{m}=1350^{\circ} \mathrm{C}$ & & & \\
Mantle creep activation energy & $Q$ & $\mathrm{~kJ} / \mathrm{mol}^{19}$ & 250 \\
Dry diabase activation energy & $Q$ & $\mathrm{~kJ} / \mathrm{mol}^{2}$ & 485 \\
Dry diabase stress exponent & $n$ & - & 4.7 \\
Dry diabase flow law pre-exponential & $A$ & $\mathrm{~s}{ }^{-1} \mathrm{~Pa}{ }^{-\mathrm{n}}$ & 8.5 \\
Wet diabase activation energy & $Q$ & $\mathrm{~kJ} / \mathrm{mol}^{-1}$ & 276 \\
Wet diabase stress exponent & $n$ & $\mathrm{~s}{ }^{-1} \mathrm{~Pa}{ }^{-\mathrm{n}}$ & 3.05 \\
Wet diabase flow law pre-exponential & $A$ & - & $6.12 \times 10^{-2}$ \\
\hline
\end{tabular}

value for convective instability. This critical value is determined from recent scaling relationships for the onset of convection in a fluid with strongly temperature-dependent viscosity cooled from above [Zaranek and Parmentier, 2004].

[11] Prior to the onset of convection, the temperature over the entire depth of the mantle is determined by transient heat conduction

$$
\rho c_{p} \frac{\partial T}{\partial t}=\frac{\partial}{r^{2} \partial r}\left(r^{2} k \frac{\partial T}{\partial r}\right)+H
$$

where $r$ is radial distance from the center of the planet.

[12] After the onset of convection, temperatures in the conductive lid are determined by solutions of (6) in a conductive lid of thickness $L$. Solutions in the lid are determined by prescribing the surface temperature at $r=R_{s}$, and the heat flux on $r=R_{s}-L$. With an increase (decrease) in lid thickness $d L$ over a small time interval $d t$, material of heat capacity $\rho c_{p}$ within the thermal boundary layer of thickness $\delta_{c}$ cools (heats) by an amount $-\left(\Delta T_{c} / \varepsilon_{c}\right) d L$. During the same time interval an amount of heat $f d t$ is transported to the base of the thermal boundary layer. These two additions of heat must be balanced by heat conduction into the overlying conductive lid as

$$
\rho c_{p} \Delta T_{c} \frac{d L}{d t}+f=-k \frac{\partial T}{\partial r}{ }_{r=R_{s}-L} .
$$

Simultaneously, over an increment of thickening, the temperature at the base of the conductive lid must remain equal to the temperature (or viscosity increase) at which significant convective flow ceases

$$
T_{r=R_{s}-L}=T_{m}-\Delta T_{c}
$$

[13] Temperatures within the conductive lid are described by discrete values on a grid of points uniformly spaced in depth. Spatial derivatives in equations (6), (6a), and (6b) are 

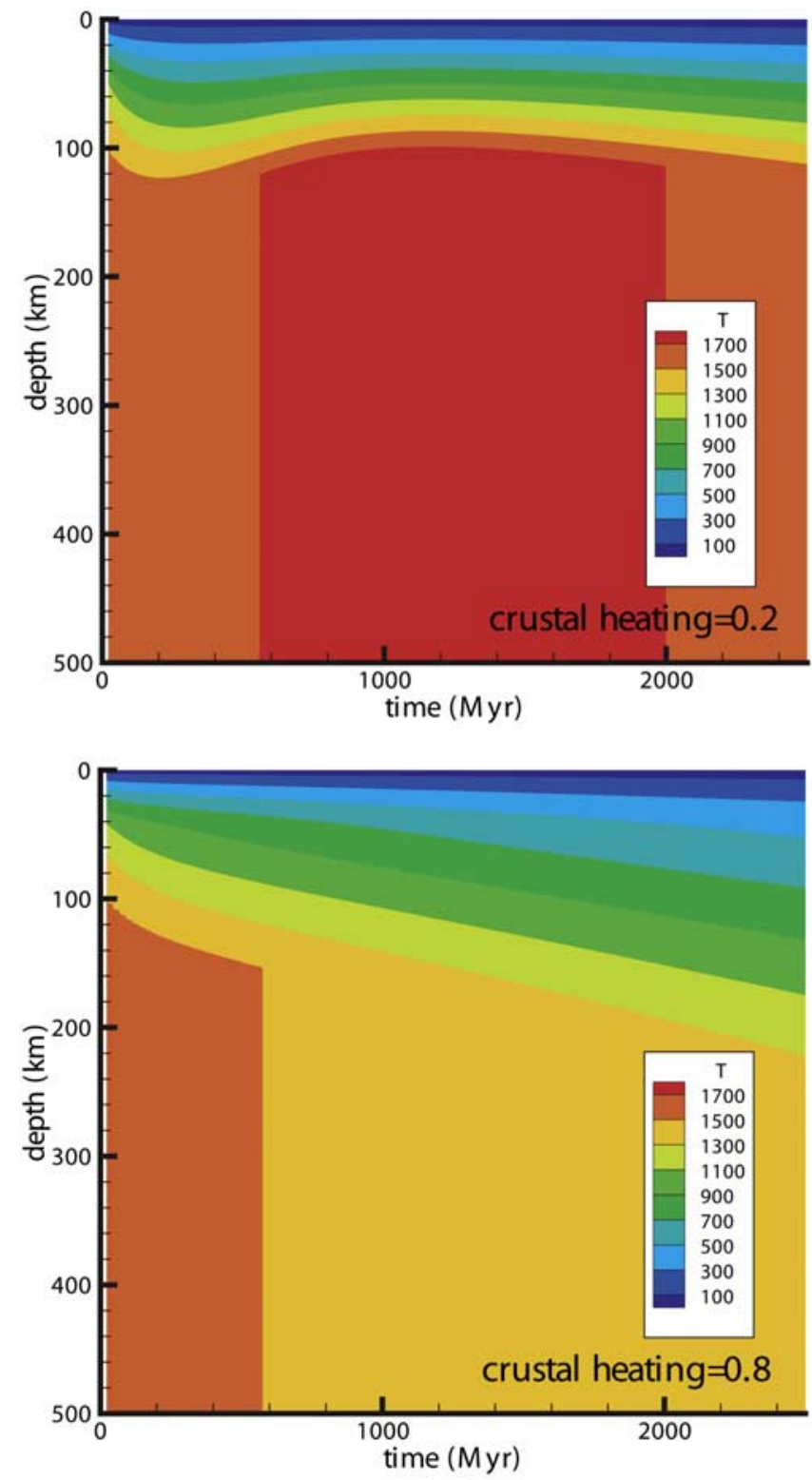

Figure 2. Mantle potential temperature as a function of depth and time for thermal models with crustal heating fractions of 0.2 and 0.8 and an initial temperature of $1500^{\circ} \mathrm{C}$. Other parameters are given in the text and Table 1. A smaller crustal heating fraction results in more mantle heating so that the mantle heats up several hundred ${ }^{\circ} \mathrm{C}$ above its initial temperature. At the higher crustal heating fraction, the mantle cools monotonically at a rate determined by thermal convection beneath a conducting lid.

approximated by central finite difference derivatives. Time derivatives in (1), (5), and (6a) are approximated by simple forward differences in time, yielding an explicit system of algebraic equations to calculate $T_{m}$ and temperatures in the conductive lid at each new time. The base of the lid at depth $L$ is allowed to vary continuously between grid points with the temperature and temperature gradient at the base in (6b) and (6b) defined by linear extrapolation from values at the two grid points just above the base of the lid.
[14] Examples of thermal evolution models based on the above formulation are shown in Figure 2 using values of physical parameters listed in Table 1. A mantle rheology with an activation energy $Q=250 \mathrm{~kJ} / \mathrm{mol}$ and a preexponential factor that would give a viscosity of $10^{19} \mathrm{~Pa}-\mathrm{S}$ at the temperature of the Earth's upper mantle $\left(\sim 1350^{\circ} \mathrm{C}\right)$ is compatible with laboratory-derived flow laws for olivine [Hirth and Kohlstedt, 1996; Mei and Kohlstedt, 2000]. Higher viscosities have the principal effect of lengthening the timescale of thermal evolution and reducing the secular cooling contribution to crustal temperatures.

[15] The distribution of heat-producing elements also affects temperatures in the crust. In the models presented here, we assume a bulk concentration of heat-producing elements in the silicate planet and explore different partitioning of heat production between the mantle and the crust. Two different abundances of heat producing elements have been suggested for Mars. The first is essentially chondritic [McDonough and Sun, 1995; Wanke and Dreibus, 1994]. A second model [Lodders and Fegley, 1997] has about a three times higher potassium abundance, and given the short $K^{40}$ decay time produces about twice the early heating rate. Hauck and Phillips [2002] provide a concise summary and discussion of the two heat production models. Given the highly heterogeneous interior that could result from the early differentiation of Mars [e.g., Elkins-Tanton et al., $2005 \mathrm{a}, 2005 \mathrm{~b}$ ] and therefore large uncertainties in the relationship of the composition of surface materials to that of the bulk interior, we have chosen the simple chondritic model. Models with higher heat production will only increase lower crustal temperatures and reinforce the conclusions of this work. $U, T h$, and $K$ values adopted in our models are given in Table 1.

[16] Long-lived radiogenic elements $U, T h$, and $K$ are highly incompatible in mantle rocks and will be strongly partitioned into melt during mantle partial melting. To a first approximation their concentration in the crust will be the initial mantle concentration divided by the degree of melting. If the degree of melting were $F$, then the fraction (crustal volume/mantle volume) $/ F$ of the mantle would have melted, and this would be the resulting overall fraction of heat production in the crust. For $F=10 \%$ and a $50 \mathrm{~km}$ thick crust, approximately $50 \%$ of the heat production of the planet would be in the crust. In our models, uniform concentration of heat producing elements with depth in the crust is assumed. In the results to follow, the fraction of heat production in the crust is varied from 0.2 to 0.8 .

[17] The physical characteristics of the early Martian crust are not well constrained by direct observations. However, the near-surface region of a planet early in its evolution should be characterized by a brecciated, porous regolith with a thermal conductivity that may be significantly lower than that of deeper crustal rock. The Lunar Highlands, which are believed to have preserved surface characteristics dating from the time of significant impact activity, may be one possible analog for very early Mars. For the Moon, returned samples in addition to gravity and seismic data are available to constrain crustal structure. Warren and Rasmussen [1987] summarized evidence for a 2- to 3-km-thick layer of brecciated, porous rock overlying a 20- to 25-km-thick layer of fractured rock. They suggested that the porous layer may be created from a thicker layer of 
impact ejecta by compaction and annealing at sufficiently high temperature (and lithostatic pressure). If so, a significant difference between regoliths of the Moon and Mars may be that greater lithostatic pressure at a given depth, due to the higher gravity of Mars, favors a thinner porous layer. Higher impact velocities due higher gravity would favor a thicker layer. On the basis of correlations of thermal conductivity and porosity, Warren and Rasmussen suggest that the thermal conductivity in the brecciated regolith is reduced by a factor of 10 from that of intact rock. In the results to follow, we will consider the effect of layers 1 and $2 \mathrm{~km}$ thick in which the thermal conductivity is reduced by a factor of 10. If the pore space were not empty but filled with a conducting material, perhaps water or water ice on Mars, the thermal conductivity would be reduced by a much smaller factor and would not be less than the conductivity of the pore-space-filling material, $2.4 \mathrm{~W} / \mathrm{m}-{ }^{\circ} \mathrm{C}$ and $0.6 \mathrm{~W} / \mathrm{m}-{ }^{\circ} \mathrm{C}$ for liquid water and water ice, respectively.

\section{Crustal Flow and Relaxation in Thickness Variations}

[18] Variations in the thickness of a low-density crust result from topography both at the surface and at the crustmantle boundary. Thermally activated creep in the crust and mantle will allow this topography and its corresponding crustal thickness variation to relax over time. Earlier studies [e.g., Solomon et al., 1982] show that topography at the surface and at the crust-mantle boundary evolve on two distinct timescales involving two different forms of deformation. Following relatively rapid, almost vertical, column-wise isostasy, longer-term horizontal flow reduces an initially nonuniform crust to the lowest potential energy state of constant thickness. This flow, illustrated in Figure 1, is driven by horizontal pressure gradients that result from isostatically compensated crustal thickness variations. Since thermally activated creep of crustal rock is strongly temperature-dependent, most of this flow must occur near the base of the crust where the temperature is highest. For conditions of interest, the mantle should flow at much lower rates than the weak crustal layer. A typical velocity profile is shown in Figure 1.

[19] As illustrated in Figure 3, crustal flow should be well approximated as locally horizontal viscous flow with a viscosity that varies with temperature or depth in the crust. The temperature, $T$, within the crust at depth $z$ is determined, on the long timescales of interest, by steady state conductive transfer of heat to the surface

$$
T=T_{s}+f_{c m}(D-z) / k+H\left(D^{2}-z^{2}\right) / 2 k,
$$

depending on the surface temperature $T_{s}$, the heat flux at the crust-mantle boundary $f_{c m}$, and the thickness of the crust $D$. This assumes a uniform thermal conductivity and does not account for affects discussed below. The horizontal velocity $u_{x}(z)$ within the crust is determined by a thermally activated creep flow law

$$
\frac{\partial u_{x}}{\partial z}=A \exp \left(-\frac{Q}{R T}\right) \sigma_{x z}^{n}=A \exp \left(-\frac{Q}{R T}\right)\left(\Delta \rho g \frac{\partial D}{\partial x}\right)^{n},
$$

where the stress exponent $n$, and preexponential factor $A$ can be determined from laboratory flow laws of candidate crustal materials. To a good approximation, flow at the base of the crust occurs primarily in a layer of thickness $h$ where the viscosity is within about a factor of ten of that at the crust-mantle boundary

$$
h \approx 2.23\left(\frac{k T_{c m}}{f_{c m}}\right)\left(\frac{R T_{c m}}{Q}\right) .
$$

The rate of change of crustal thickness $D$ is determined by the horizontal flux of crust

$$
\frac{\partial D}{\partial t}=-\frac{\partial q}{\partial x}
$$

where, with the above approximations,

$$
q=\int_{0}^{D} u_{x} d z \approx-\beta A \exp \left(-\frac{Q}{R T_{c m}}\right)\left(\Delta \rho g \frac{\partial D}{\partial x}\right)^{n} h^{n+2} .
$$

where $\beta$ is a coefficient near unity. Substituting (12) into (11) results in a single equation which can be solved to obtain $D(x, t)$ subject to suitable initial and boundary conditions. This study has the simple objective of providing only an order of magnitude estimate of the relaxation time $\tau$ of crustal thickness variations of amplitude $\Delta D$ over a horizontal scale $\Delta x$. Equation (11) can then be approximated $\Delta D / \tau \cong-\Delta q / \Delta x$ giving a crustal relaxation rate $r$

$$
r=\frac{1}{\tau}=\frac{n \beta A \exp \left(-\frac{Q}{R T_{c m}}\right)(\Delta \rho g)^{n} h^{n+2}\left(\frac{\Delta D}{\Delta x}\right)^{n-1}}{\Delta x^{2}} .
$$

[20] Crustal relaxation rate as a function of time for the thermal evolution models described above, with $\Delta D=10 \mathrm{~km}$ and $\Delta x=500 \mathrm{~km}$, are shown in Figure 3. Here the wet and dry diabase flow laws given by Caristan [1982] and Mackwell et al. [1998], respectively, have been adopted. Mackwell et al. point out that the Caristan experiments may have resulted in partial melting of the samples producing an overly weak rheology. However, the partial melt fraction seems likely to have been too small to significantly affect the rheology. The specific mineralogy may be less important since flow laws for wet quartzite [Hirth et al., 2001] and wet anorthite [Rybacki and Dresen, 2004] give relaxation rates of the same order of magnitude as the wet diabase flow law.

[21] If Mars retained water as it accreted, the mantle was likely to be wet during the early evolution, with significant amounts of water dissolved in nominally anhydrous mantle minerals. Water, like heat-producing $U, T h$, and $K$, is relatively incompatible in mantle minerals and would be strongly partitioned in to the liquid phase during mantle melting. The lower crust of Mars, as a product of mantle partial melting, is likely to be enriched in elements incompatible in the residual mantle, including water. In a planet with substantial evidence for the presence of water on the surface, a wet rheology for the lower crust seems preferred. However, if water were not retained during accretion but was added as a "late veneer" [Dreibus and Wanke, 1987], 
then the mantle of Mars and crust generated by its partial melting would be dry. Melt inclusions from the meteorites that crystallized at some depth contain amphiboles, implying a significant initial magmatic water content. McSween et al. [2001] argue from phase equilibrium considerations that the shergottite parent magmas contained $\sim 2 \mathrm{wt} \%$ water. $\mathrm{D} / \mathrm{H}$ ratios in SNC minerals are also significantly below atmospheric values suggesting the presence of primordial water [Boctor et al., 2003]. More recently, Medard and
Grove [2006] also argue for petrologically significant amounts of water in the early Martian mantle. On the basis of indigenous water contents of the meteorite, Jones [2004] estimates a much smaller water contents of the mantle source $\sim 350 \mathrm{ppm}$ for Chassigny. Thus no general consensus has emerged on the initial water content of the Martian mantle. However, distinguishing between petrologically and rheologically significant water contents is important. Petrologically significant amounts of water are at the level of a
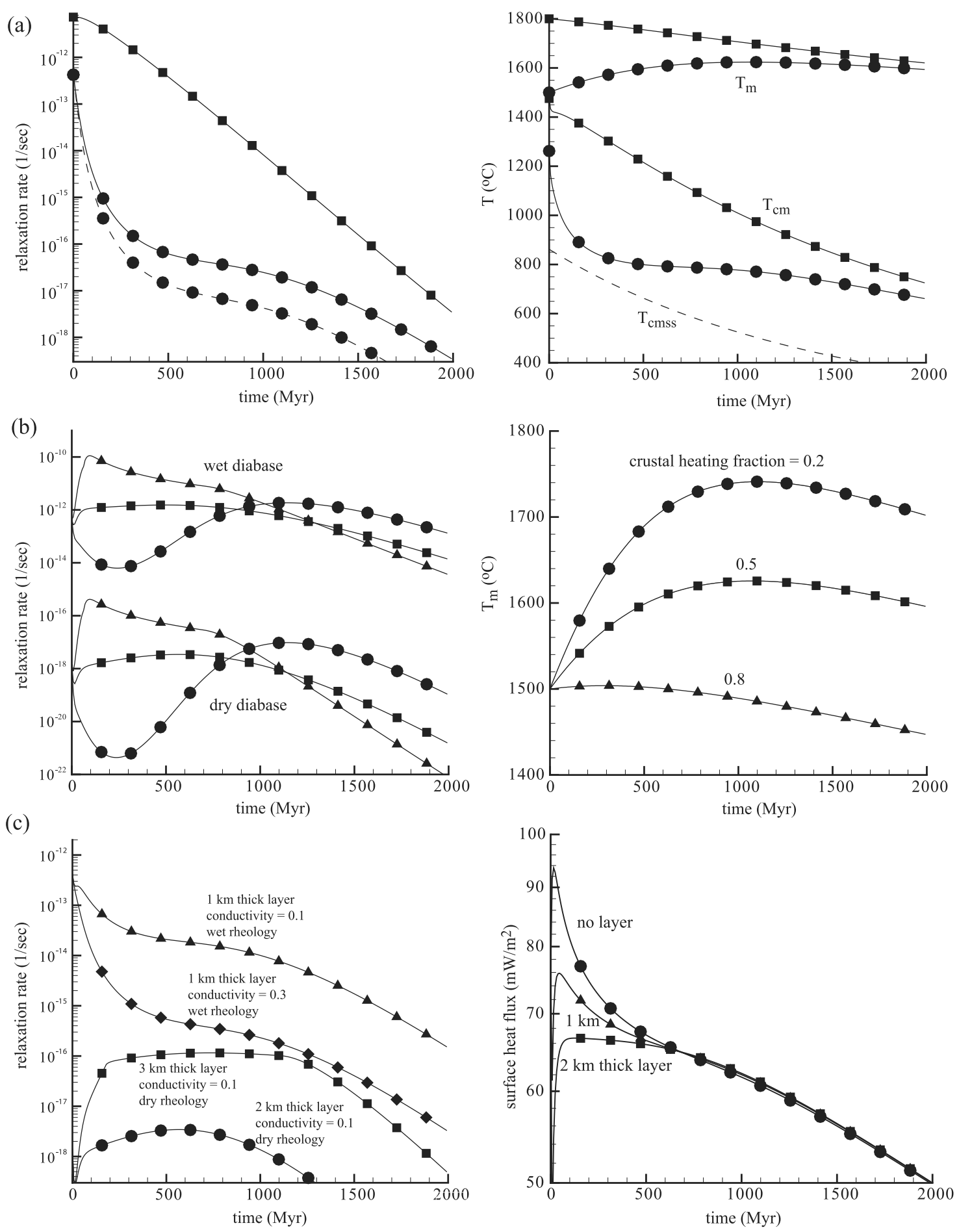

Figure 3 
few weight percent. In olivine, for example, water significantly reduces the flow stress at concentrations of only a few hundred ppm. Not much water is needed to be rheologically significant.

\section{Thermal Evolution and Crustal Relaxation}

[22] Potential temperatures calculated from convective thermal evolution models are shown in Figure 3a for initial temperatures of $1500^{\circ}$ and $1800^{\circ} \mathrm{C}$ as a function of time, both with a crustal heating fraction of 0.5 . For the higher initial temperature, the mantle cools monotonically initially at a relatively slow rate while for the lower initial temperature, radioactive heating exceeds secular cooling over the first several Gyr of evolution and the interior heats up. The crust-mantle boundary temperature $T_{c m}$ is also shown for comparison, with the temperature calculated for a steady state thermal evolution. Secular cooling of the mantle contributes significantly to $T_{c m}$ in both cases, but more for the higher initial temperature. Relaxation rates for a wet diabase rheology, also shown for these two cases. A relaxation rate less than $10^{-17} \mathrm{~s}^{-1}$ would be required to preserve crustal thickness variations produced during the first 750 Myr. For both cases, the relaxation rate during the first Gyr is large enough that crustal thickness variations generated very early in the evolution should relax.

[23] Figure $3 \mathrm{~b}$ shows interior temperature and relaxation rates as a function of time for crustal heating fractions 0.2 , 0.5 , and 0.8 , all with a $1500^{\circ} \mathrm{C}$ initial mantle potential temperature. The crustal heating fraction 0.5 is common to Figures $3 \mathrm{a}$ and $3 \mathrm{~b}$. For a low crustal heating fraction (high mantle heating rate), the interior heats above its initial temperature while for high crustal heating the interior cools monotonically. A higher crustal heating fraction results in a higher relaxation rate. The relaxation rate is given for both wet and dry diabase rheology. All cases shown in Figures $3 b$ include a 2-km-thick dry brecciated surface layer in which the conductivity is reduced by a factor 0.1 . The dry rheology would preserve crustal thickness variation even in the presence of the 2-km-thick low-conductivity layer, except for the highest crustal heating. For the wet diabase rheology crustal thickness variations rapidly relax even for the lowest crustal heating fraction.

[24] Figure 3c shows relaxation rate for both wet and dry diabase rheology with a range of low-conductivity layer thicknesses up to $3 \mathrm{~km}$ with several values for the thermal conductivity reduction. The relaxation rate increases with increasing thickness or decreasing conductivity of the lowconductivity layer. The case with no layer would approximate a brecciated crustal layer in which the pore space is filled with water ice. The smaller conductivity reduction (0.3) would represent water-filled pore space. For the wet rheology, neither case comes close to preserving crustal thickness variations over the first Gyr For the dry rheology, a 2-km-thick layer would preserve Noachian age crustal thickness variations, but a 3-km-thick layer would not.

[25] Figure 3c also shows surface heat flow as a function of time. The presence of a crustal low-conductivity layer reduces surface heat flow, but only in the early evolution. During the early evolution, even in the presence of a lowconductivity crustal layer, surface heat flow exceeds $60 \mathrm{~mW} / \mathrm{m}^{2}$. Studies of the elastic support of topography [McGovern et al., 2002, 2004], from gravity anomalies associated with Noachian age topographic features shows that they are almost indistinguishable from being isostatically supported. Minimum estimates of surface heat flow inferred from the small amount of elastic support allowed are as large as $50 \mathrm{~mW} / \mathrm{m}^{2}$ [McGovern et al., 2004] (Table 1), comparable to values calculated in the models of Figure 3c. However, by the end of the Hesperian or beginning of the Amazonian ( $1500 \mathrm{Myr})$, elastic thicknesses appear to require heat flows not larger than about $30 \mathrm{~mW} / \mathrm{m}^{2}$. This is lower than values predicted both by the models in Figure $3 \mathrm{c}$ and the nominal model of Hauck and Phillips [2002, Figure 2d]. This discrepancy needs further study but may be related to thermal structure of the crust and upper mantle. The relationship of elastic thickness and heat flow [e.g., Solomon and Head, 1990] employed by McGovern et al. was based on a linear thermal gradient through the lithosphere. Understanding how more realistic temperature distributions in the crust and uppermost mantle might affect the elastic thickness-heat flow relationship

\footnotetext{
Figure 3. (a) (left) Relaxation rate of crustal thickness variations and (right) mantle potential temperature $T_{m}$ and crustmantle boundary temperatures $T_{c m}$ as a function of time. Examples are shown for initial temperatures $1500^{\circ}$ and $1800^{\circ} \mathrm{C}$, denoted by circles and squares, respectively, both with a crustal heating fraction 0.5. Dashed curve on the right shows $T_{c m}$ for a steady state thermal model. Relaxation rates $<10^{-17} \mathrm{~s}^{-1}$ are required to preserve crustal thickness variations for Gyr timescales. Relaxation rates for a wet diabase rheology and no crustal low-conductivity layer exceed this value even for the case with cooler initial mantle temperature. Solid curves on the left are for a $273^{\circ} \mathrm{K}$ surface temperature. Dashed curve on the left shows the relaxation rate with a lower $220^{\circ} \mathrm{K}$ surface temperature. (b) Thermal evolution models with a $1500^{\circ} \mathrm{C}$ initial temperature for crustal heating fractions of 0.2 (circles), 0.5 (squares), and 0.8 (triangles) with a 2-km-thick, lowconductivity (brecciated) crustal layer in which the conductivity is reduced to 0.1 that of unfractured crust. Crustal relaxation rates are significantly enhanced by the presence of the low-conductivity layer, as can be seen by comparing wet diabase relaxation rates with those given in Figure 3a. Even with a low-conductivity layer, relaxation rates for the dry diabase rheology are low enough to allow the preservation of crustal thickness variations, except for the high crustal heating fraction. (c) (left) Relaxation rate and (right) surface heat flow as functions of time for a model with a crustal heating fraction 0.5 , initial temperature $1500^{\circ} \mathrm{C}$. Relaxation rates are shown for several low-conductivity layer thicknesses and for both wet and dry rheology. For wet rheology, relaxation rates even allowing higher conductivity in the low-conductivity layer the would not allow the preservation of topography. For the dry rheology, a low-conductivity layer $2 \mathrm{~km}$ thick would allow the preservation of crustal thickness variations, but a 3-km-thick layer would not. Heat flow on the right is shown for low-conductivity layer of thicknesses 0 (circles), $1 \mathrm{~km}$ (triangles), and $2 \mathrm{~km}$ (squares) with conductivity reduced by a factor 0.1 in all cases.
} 
would be of interest but is beyond the scope the present study.

\section{Hydrothermal Cooling of the Crust}

[26] A fractured permeable regolith, if water saturated, could conceivably allow significant hydrothermal convective cooling of the crust. This possibility is well worth considering for Mars because the permeability required is not large and there is good reason to believe that the planet had significant amounts of water at least in the shallow crust for much of its geological history. The Martian surface shows pervasive evidence of Noachian-aged surface water exhibited in the geology [cf. Carr, 1996], and neutron flux and gamma-ray maps show evidence for present-day hydrogen concentrations indicative of substantial water in the top meter of the subsurface [Feldman et al., 2002; Mitrofanov et al., 2002]. It has been hypothesized that the remnants of an early Martian ocean are preserved within the brecciated crust [Clifford and Parker, 2001], perhaps to significant depths in the crustal column [Schrag and Zuber, 2003].

[27] A deeper, fractured layer should be present beneath the brecciated uppermost crust. The porosity of this deeper fractured layer may be small enough that its thermal conductivity would not be significantly affected. However, even small porosity in well-connected fracture systems with fractures spaced at the meter scale or smaller [see Hanna and Phillips, 2005] can create significant levels of permeability. On the Moon, low seismic velocities in the upper $25 \mathrm{~km}$ of the crust [Goins et al., 1981; Khan and Mosegaard, 2000, 2001] is thought to reflect fractured rock [Simmons et al., 1973] with a porosity of about $1 \%$. An impact-fractured layer should also have been present on Mars, perhaps with a similar porosity to that on the Moon. If the bottom of such a permeable layer is determined by the closing of fractures under lithostatic stress, the layer thickness on Mars should be about one third that on the Moon. While, greater lithostatic pressure would tend to close fractures in a fractured layer at shallower depths on Mars than on the Moon, impacting bodies with higher kinetic energy on Mars could cause fracturing to greater depth, so that the 20- to $25-\mathrm{km}$-thick layer of fracturing on the Moon could extend to even greater depths on Mars. Given evidence of the important role of groundwater on Mars, several models for the hydrologic properties of the Martian crust have been proposed [MacKinnon and Tanaka, 1989; Clifford, 1993; Hanna and Phillips, 2005]. Hanna and Phillips [2005] provide a good summary of earlier models. They also conclude that the permeability of the crust should be dominated by fractures, with a small permeability due to impact brecciation. At depths greater than about $4 \mathrm{~km}$, they estimate a fracture permeability of $\sim 10^{-15} \mathrm{~m}^{2}$. They suggest that the depth to which fractures provide permeability is limited by the brittle-plastic transition and that this would extend to depths of $20-25 \mathrm{~km}$ on Mars. Hydrothermally altered minerals may fill cracks, reducing permeability. Hydrothermal cooling may thus decrease as the planet evolves and could be most effective when impacts are frequent enough to continually generate new fractures.

[28] The rate of hydrothermal cooling is determined by a Rayleigh number that depends on the product of the permeability and layer thickness and on the physical properties of water

$$
R a=\frac{\rho \alpha \Delta T g K D_{h}}{\mu \kappa} .
$$

where $\rho, \alpha$, and $\mu$ are the density, thermal expansion coefficient, and viscosity of the fluid (water) respectively; $\kappa$, $K$, and $D_{h}$ are the thermal diffusivity, permeability, and thickness of the fluid saturated rock layer. The heat flux $f$ through the permeable layer can be expressed in terms of the ratio of hydrothermal convective heat transfer to purely conductive heat flux or Nusselt number, $N u$, as

$$
f=N u k\left(\frac{T_{b}-T_{s}}{D_{h}}\right),
$$

where $T_{b}$ is the temperature at the bottom of the layer of thickness $D_{h}$. Laboratory experiments [Elder, 1967] give

$$
N u=0.025 R a,
$$

where in $R a, \Delta T=T_{b}-T_{s}$. Similar results have been obtained from numerical experiments [cf. Combarnous, 1978]. With a permeability $K \sim 10^{-15} \mathrm{~m}^{2}$, as estimated by Hanna and Phillips [2005], $T_{b}-T_{s} \sim 300^{\circ} \mathrm{C}, D_{h} \sim 20 \mathrm{~km}$, and appropriate values for physical properties of water or water saturated rock, give $R a \sim 100$. Then equation (16) gives $N u \sim 2-3$.

[29] Figure 4 shows the relaxation rate of crustal thickness variations as a function of time for several $N u$ values with two layer thicknesses. The mantle thermal evolution model here with an initial mantle temperature of $1500^{\circ} \mathrm{C}$ and 0.5 crustal heating fraction corresponds to one of those shown in Figure 3. The case with $N u=1$ corresponds to no hydrothermal cooling; and as discussed in the context of Figure 3, the relaxation rate exceeds $10^{-17} \mathrm{~s}^{-1}$ during the first Gyr of evolution. For $N u=2$ in a permeable layer only $10 \mathrm{~km}$ thick, the relaxation rate is small enough to preserve crustal thickness variations of early Noachian age with the wet diabase crustal rheology.

[30] An interesting speculation is that hydrothermal alteration may also explain the difference in the intensity of magnetic anomalies between the southern highlands and northern plains [cf. Stevenson, 2001; Solomon et al., 2005]. If the same crustal mineralogy is responsible for remnant magnetization and if hydrothermal alteration extends to the same depth in both the highlands and the lowland plains, then thicker crust in the highlands would result in a thicker, unaltered, still magnetized layer.

\section{Stable Stratification of a Differentiated Mantle}

[31] Magma ocean fractional solidification is expected to generate a highly unstable density stratification [ElkinsTanton et al., 2003a, 2003b, 2005a]. Depending on the rate of thermally activated creep, this will overturn to a strongly stable stratification in times as short at 1-10 Myr. Decompression melting during this overturn may create an early crust [Elkins-Tanton et al., 2005b]. The stable stratification following mantle overturn could be sufficient to suppress solid-state thermal convection so that the mantle would cool 

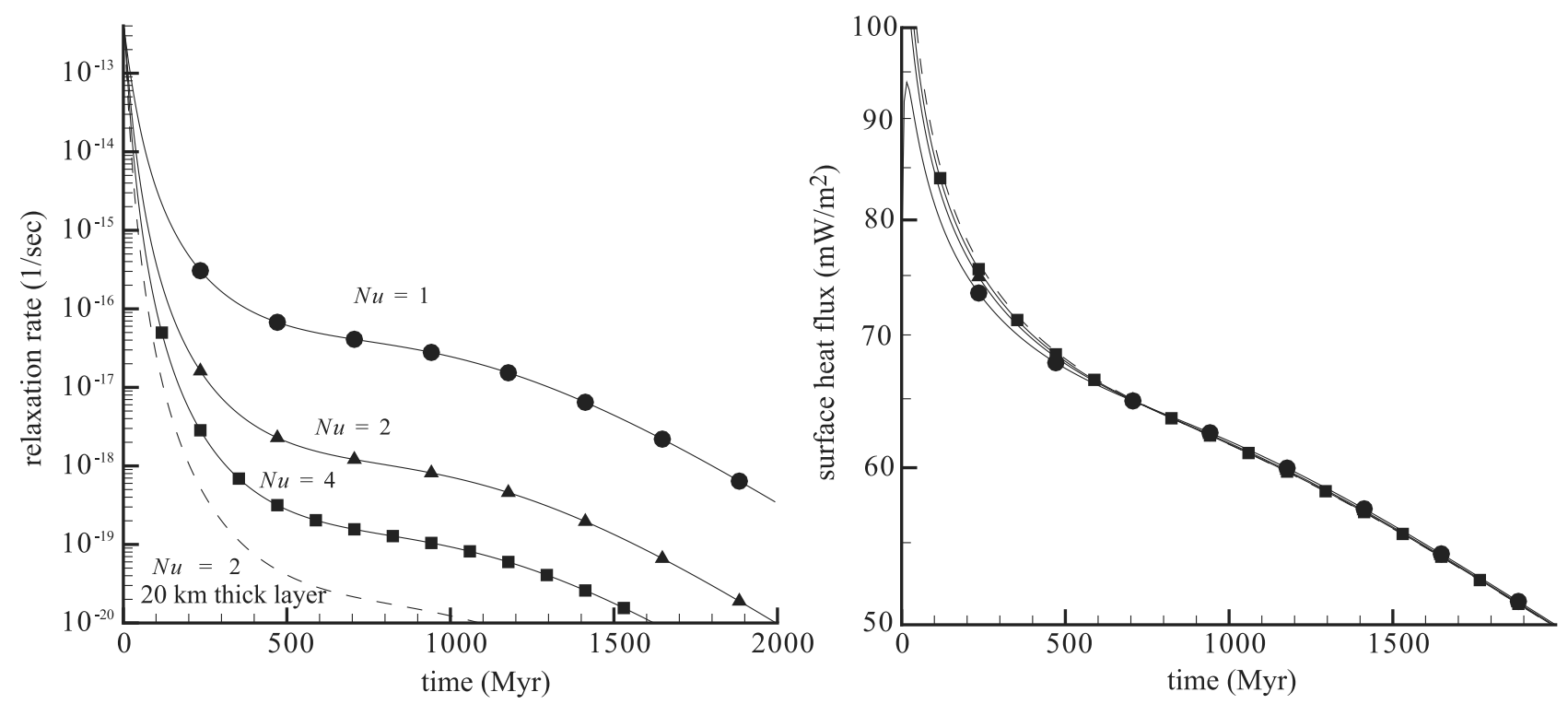

Figure 4. (left) Crustal relaxation rate with the wet diabase rheology and (right) surface heat flow as functions of time for a thermal evolution with $1500^{\circ} \mathrm{C}$ initial temperature and 0.5 crustal heating fraction. The effect of a 10-km-thick hydrothermally cooled layer is shown for hydrothermal Nusselt numbers $(N u)$ of 1 (circles), 2 (triangles), and 4 (squares). Dashed curve is for a 20-km-thick layer with $N u=2$. Hydrothermal cooling with $N u \approx 2-4$ in a $10-\mathrm{km}$-thick layer would allow the preservation of crustal thickness variations even with the wet diabase rheology. Surface heat flow is not strongly affected by the presence of a hydrothermally cooled layer.
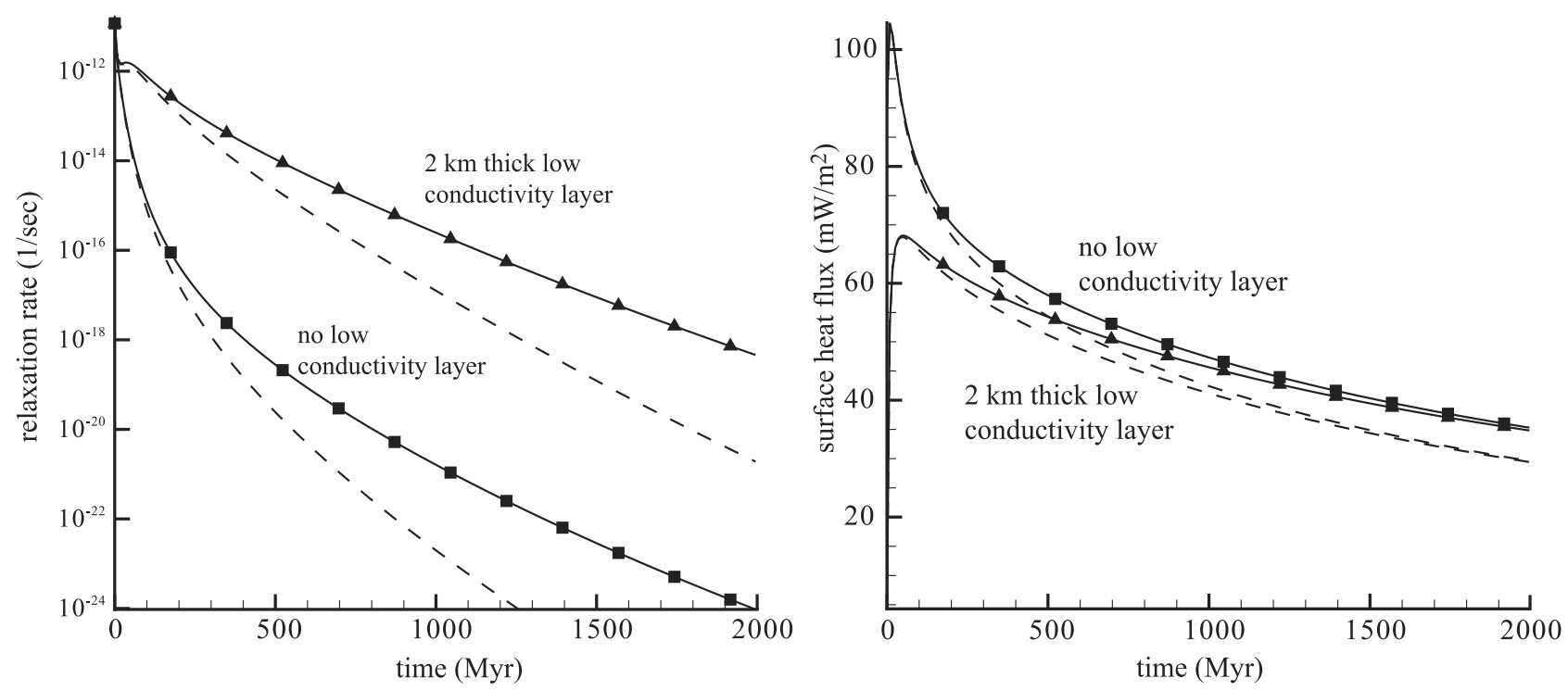

Figure 5. (left) Crustal relaxation rate for wet diabase rheology and (right) surface heat flow as functions of time for a model with mantle cooling solely by heat conduction from an initial temperature $1500^{\circ} \mathrm{C}$ with crustal heating fraction 0.5 . Cases with a 2-km-thick low-conductivity layer $(0.1$ of normal crustal conductivity) and no low-conductivity layer are shown by circles and squares, respectively. The dashed curves show the case of a differentiated mantle in which mantle radiogenic heat sources do not contribute to crustal temperatures; see text for more discussion. With a dry brecciated low-conductivity layer, relaxation rates are too high to allow preservation of crustal thickness variations. A differentiated mantle reduces relaxation rates, but not sufficiently to preserve crustal thickness variations. The case with no crustal low-conductivity layer appears to have a low enough relaxation rate to preserve a significant fraction of late Noachian-aged crustal thickness variations. 
at a slower rate controlled by heat conduction. In the fractional solidification model, mantle heat production would be concentrated in the last mantle to solidify, which ends up at the bottom of an overturned mantle. This heating may eventually lead to thermal convective instability [cf. Alley and Parmentier, 1998]; but early in the evolution, this concentration of incompatible elements at depth in a stably stratified mantle would isolate mantle heat sources from the crust. Figure 5 shows the consequences of a stable mantle stratification on crustal relaxation for the case that radiogenic heat sources remain uniformly distributed in the mantle and for the case that these heat sources reside at the bottom of the mantle and do not contribute to crustal temperatures.

\section{Summary}

[32] MGS gravity and MOLA topography show that crustal thickness variations have persisted since the earliest evolution of planet even though the elastic thickness beneath early structural/topographic features was small. Thermal evolution models in which the mantle loses heat by solid-state thermal convection indicate a significant contribution of secular mantle cooling to lower crustal temperatures, with temperatures significantly higher than for a steady state thermal evolution. The models indicate that creep in a lower crust with a dry diabase rheology would be sufficiently slow to allow the preservation of crustal thickness variations. However, temperatures in the lower crust are sufficiently high that a wet-diabase rheology would have allowed crustal thickness variations created in the Noachian to have relaxed.

[33] The presence of an impact-brecciated crustal layer with low thermal conductivity, if comparable to that thought to be present on the Moon, would significantly increase lower crustal temperatures. With a brecciated crustal layer comparable to that expected for the Moon ( $\sim 2 \mathrm{~km}$ thick), very rapid relaxation of crustal thickness variations is predicted. Even a dry diabase rheology does not allow the preservation of crustal thickness variations if a dry impactbrecciated layer more than $3 \mathrm{~km}$ thick were present. A water saturated brecciated layer would have a thermal conductivity only slightly less than intact rock and would not significantly increase crustal temperatures.

[34] The cooler crust required to preserve crustal thickness variations for the wet crustal rheology may be a consequence of (1) a stably stratified mantle that does not convect thermally and in which heat sources are fractionated into compositionally dense mantle at depth or (2) hydrothermal heat transfer in the a fractured portion of the upper crust. A conductively cooling differentiated mantle would allow the preservation of crustal thickness variations so long as the brecciated low-conductivity layer were thin, probably less than $1 \mathrm{~km}$. Hydrothermal cooling of the upper $10 \mathrm{~km}$ of the crust can explain both preservation of crustal thickness variations and the inferred elastic thickness, so long as convective heat transfer rates are not too large.

\section{References}

Alley, K. M., and E. M. Parmentier (1998), Numerical experiments on thermal convection in a chemically stratified viscous fluid heated from below: Implications for a model of lunar evolution, Phys. Earth Planet Inter., 108, 15-32.
Boctor, N. Z., C. M. O. Alexander, J. Wang, and E. Hauri (2003), The sources of water in Martian meteorites: Clues from hydrogen isotopes, Geochim. Cosmochim. Acta, 67, 3971-3989.

Caristan, Y. L. (1982), The transition from high temperature creep to fracture in Maryland diabase, J. Geophys. Res., 87, 6781-6790.

Carr, M. H. (1996), Water on Mars, Oxford Univ. Press, New York.

Choblet, G., O. Grasset, E. M. Parmentier, and C. Sotin (1999), Mars thermal evolution revisited, Lunar Planet. Sci. Conf., XXX, 1556-1557.

Clifford, S. M. (1993), A model for the hydrologic and climatic behavior on Mars, J. Geophys. Res., 98, 10,973-11,016.

Clifford, S. M., and T. L. Parker (2001), The evolution of the Martian hydrosphere: Implications for the fate of a primordial ocean and the current state of the northern plains, Icarus, 154, 40-79.

Combarnous, M. (1978), Natural convection in porous media and geothermal systems, Int. Heat Transfer Conf., 6, 45-59.

Davaille, A., and C. Jaupart (1993), Transient high-Rayleigh-number thermal convection with large viscosity variations, J. Fluid Mech., 253, $141-166$.

Dreibus, G., and H. Wanke (1987), Volatiles on Earth and Mars: A comparison, Icarus, 71, 225-241.

Elder, J. W. (1967), Steady free convection in a porous medium heated from below, J. Fluid Mech., 27, 29-48.

Elkins-Tanton, L. T., E. M. Parmentier, and P. C. Hess (2003a), A model for Martian magma ocean crystallization and overturn, Lunar Planet. Sci. Conf. [CD-ROM], XXXIV, Abstract 1479.

Elkins-Tanton, L. T., E. M. Parmentier, and P. C. Hess (2003b), Magma ocean fractional crystallization and cumulate overturn in terrestrial planets: Implications for Mars, Meteorol. Planet. Sci., 38, 1753-1771.

Elkins-Tanton, L. T., S. E. Zaranek, E. M. Parmentier, and P. C. Hess (2005a), Early magnetic field and magmatic activity on Mars from magma ocean cumulate overturn, Earth Planet. Sci. Lett., 236, 1-12.

Elkins-Tanton, L. T., P. C. Hess, and E. M. Parmentier (2005b), Possible formation of ancient crust on Mars through magma ocean processes, J. Geophys. Res., 110, E12S01, doi:10.1029/2005JE002480.

Feldman, W. C., et al. (2002), Global distribution of neutrons from Mars: Results from Mars Odyssey, Science, 297, 75-78.

Goins, N. R., A. M. Dainty, and M. N. Toksoz (1981), Structure of the lunar crust at Highland site Apollo station 16, Geophys. Res. Lett., 8, 29-32.

Grasset, O., and E. M. Parmentier (1998), Thermal convection in a volumetrically heated, infinite Prandtl number fluid with strongly temperature-dependent viscosity: Implications for planetary thermal evolution, J. Geophys. Res., 103, 18,171-18,181.

Hanna, J. C., and R. J. Phillips (2005), Hydrological modeling of the Martian crust with application to the pressurization of aquifers, J. Geophys. Res., 110, E01004, doi:10.1029/2004JE002330.

Hauck, S. A., II, and R. J. Phillips (2002), Thermal and crustal evolution of Mars, J. Geophys. Res., 107(E7), 5052, doi:10.1029/2001JE001801.

Hirth, G., and D. L. Kohlstedt (1996), Water in the oceanic upper mantle: Implications for rheology, melt extraction, and the evolution of the lithosphere, Earth Planet. Sci. Lett., 144, 93-108.

Hirth, G., C. Teyssier, and W. J. Dunlap (2001), An evaluation of quartzite flow laws based on comparisons between experimentally and naturally deformed rocks, Int. J. Earth Sci., 90, 77-87.

Jones, J. H. (2004), The edge of wetness: The case for dry magmatism on Mars, Lunar Planet. Sci. Conf. [CD-ROM], XXXV, Abstract 1798.

Khan, A., and K. Mosegaard (2000), A new seismic velocity model of the Moon from a Monte Carlo inversion of Apollo lunar seismic data, Geophys. Res. Lett., 27, 1591-1594.

Khan, A., and K. Mosegaard (2001), New information on the deep lunar interior from an inversion of lunar free oscillation periods, Geophys. Res. Lett., 28, 1791-1794.

Lodders, K., and B. Fegley (1997), An oxygen isotope model for the composition of Mars, Icarus, 126, 394-3763.

MacKinnon, D. J., and K. L. Tanaka (1989), The impact generated Martian crust: Structure, hydrology and some geologic implications, J. Geophys. Res., 94, 17,359-17,370.

Mackwell, S. J., M. E. Zimmerman, and D. L. Kohlstedt (1998), High temperature deformation of dry diabase with application to tectonics on Venus, J. Geophys. Res., 103, 975-984.

McDonough, W. F., and S.-S. Sun (1995), The composition of the Earth, Chem. Geol., 120, 223-253.

McGovern, P. J., S. C. Solomon, D. E. Smith, M. T. Zuber, M. Simons, M. A. Wieczorek, R. J. Phillips, G. A. Neumann, O. Aharonson, and J. W. Head (2002), Localized gravity/topography admittance and correlation spectra on Mars: Implications for regional and global evolution, J. Geophys. Res., 107(E12), 5136, doi:10.1029/2002JE001854.

McGovern, P. J., S. C. Solomon, D. E. Smith, M. T. Zuber, M. Simons, M. A. Wieczorek, R. J. Phillips, G. A. Neumann, O. Aharonson, and J. W. Head (2004), Correction to "Localized gravity/topography admittance and correlation spectra on Mars: Implications for regional 
and global evolution", J. Geophys. Res., 109, E07007, doi:10.1029/ 2004JE002286.

McKenzie, D., D. N. Barnett, and D.-N. Yuan (2002), The relationship between Martian gravity and topography, Earth Planet. Sci. Lett., 195, $1-16$.

McSween, H. Y., Jr., T. L. Grove, R. C. F. Lentz, J. C. Dann, A. H. Holzheid, L. R. Riciputi, and J. G. Ryan (2001), Geochemical evidence for magmatic water within Mars from pyroxenes in the Shergotty meteorite, Nature, 409, 487-490.

Medard, E., and T. L. Grove (2006), Early hydrous melting and degassing of the Martian interior, J. Geophys. Res., 111, E11003, doi:10.1029/ 2006JE002742.

Mei, S., and D. L. Kohlstedt (2000), Influence of water on plastic deformation of olivine aggregates: 1. Diffusion creep regime, J. Geophys. Res., $105,21,457-21,469$.

Mitrofanov, I., et al. (2002), Maps of subsurface hydrogen from the highenergy neutron detector, Mars Odyssey, Science, 297, 78-81.

Neumann, G. A., M. T. Zuber, M. A. Wiezcorek, P. J. McGovern, F. G. Lemoine, and D. E. Smith (2004), Crustal structure of Mars from gravity and topography, J. Geophys. Res., 109, E08002, doi:10.1029/ 2004JE002262.

Nimmo, F., and D. J. Stevenson (2000), Influence of early plate tectonics on the thermal evolution and magnetic field of Mars, J. Geophys. Res., 105, $11,969-11,979$.

Nimmo, F., and D. J. Stevenson (2001), Estimates of Martian crustal thickness from viscous relaxation of topography, J. Geophys. Res., 106 5085-5098.

Parmentier, E. M., and M. T. Zuber (2001), Relaxation of crustal thickness variations on Mars: Implications for thermal evolution, Lunar Planet. Sci. Conf. [CD-ROM], XXXII, Abstract 1357.

Parmentier, E. M., and M. T. Zuber (2002), Preservation of crustal thickness variations on Mars: Mantle compositional stratification or hydrothermal crustal cooling?, Lunar Planet. Sci. Conf. [CD-ROM], XXXIII, Abstract 1737.

Phillips, R. J., et al. (2001), Ancient geodynamics and global-scale hydrology on Mars, Science, 291, 2587-2591.

Reese, C. C., V. S. Solomatov, J. R. Baumgardiner, and W.-S. Wang (1999), Stagnant lid convection in a spherical shell, Phys. Earth Planet. Inter. $116,1-7$.

Rybacki, E., and G. Dresen (2004), Deformation mechanism maps for feldspar rocks, Tectonophysics, 382, 173-187.
Schrag, D. P., and M. T. Zuber (2003), The carbon cycle, climate variability, and the fate of an early Martian ocean, paper presented at Sixth International Conference on Mars, Univ. of Calif., Pasadena. (Available at http:// www.lpi.usra.edu/meetings/sixthmars2003/pdf/3113.pdf.)

Simmons, G., T. Todd, and H. Wang (1973), The 25-km discontinuity: Implications for lunar history, Science, 182, 158-161.

Solomatov, V. S., and L.-N. Moresi (2000), Scaling of time-dependent stagnant lid convection: Application to small-scale convection on the Earth and other terrestrial planets, J. Geophys. Res., 105, 21,795-21,818.

Solomon, S. C., and J. W. Head (1990), Heterogeneities in the thickness of the elastic lithosphere of Mars: Constraints on heat flow and internal dynamics, J. Geophys. Res., 95, 11,073-11,083.

Solomon, S. C., R. P. Comer, and J. W. Head (1982), The evolution of impact basins: Viscous relaxation of topographic relief, J. Geophys. Res., 87, 3975-3992.

Solomon, S. C., et al. (2005), New perspectives on ancient Mars, Science, 307, $1214-1220$.

Stevenson, D. J. (2001), Mars' core and magnetism, Nature, 412, 214-291.

Wanke, H., and G. Dreibus (1994), Chemistry and accretion of Mars, Philos. Trans. R. Soc., Ser. A, 349, 2134-2137.

Warren, P. H., and K. L. Rasmussen (1987), Megaregolith insulation, internal temperatures, and bulk Uranium content of the Moon, J. Geophys. Res., 92, 3453-3465.

Zaranek, S. E., and E. M. Parmentier (2002), Convective cooling of an initially stably stratified fluid: Implications for Martian planetary evolution, Lunar Planet. Sci. Conf. [CD-ROM], XXXIII, Abstract 1580.

Zaranek, S. E., and E. M. Parmentier (2004), Convective instability of a fluid with temperature-dependent viscosity cooled from above, Earth Planet. Sci. Lett., 224, 371-386.

Zuber, M. T., et al. (2000), Internal structure and early thermal evolution of Mars from Mars Global Surveyor topography and gravity, Science, 287, $1788-1793$

E. M. Parmentier, Department of Geological Sciences, Brown University, Box 1846, Providence, RI 02912, USA. (em_parmentier@brown.edu)

M. T. Zuber, Department of Earth, Atmospheric, and Planetary Sciences, Massachusetts Institute of Technology, Cambridge, MA 02139, USA. 